\title{
A microscopic approach based on particle-vibration coupling: application to charge-exchange transitions and multiplets in odd nuclei
}

\author{
Gianluca Colò ${ }^{1,2, a}$, Yifei $\mathrm{Niu}^{2}$, Enrico Vigezzi², and Pier Francesco Bortignon ${ }^{1,2}$ \\ ${ }^{1}$ Dipartimento di Fisica, Università degli Studi di Milano, Via Celoria 16, 20133 Milano, Italy \\ ${ }^{2}$ INFN, Sezione di Milano, via Celoria 16, 20133 Milano, Italy
}

\begin{abstract}
In this contribution, we shall describe a formalism that goes beyond the simple time-dependent mean field and is based on particle-vibration coupling (PVC). Such a formalism has been developed with the idea of being self-consistent. It makes use of Skyrme effective forces, and has been used for several applications. We will focus on charge-exchange transitions, namely we will show that our model describes well both the Gamow-Teller giant resonance width, and the low-lying transitions associated with $\beta$-decay. In this latter case, including PVC produces a significant improvement of the half-lives obtained at mean-field level, and leads to a good agreement with experimental data. We will end by discussing particle-phonon multiplets in odd nuclei.
\end{abstract}

\section{Introduction}

While there is a tremendous progress in developing ab initio methods in nuclear structure theory, they are still not applicable to heavy nuclei and/or highly excited states; Density Functional Theory (DFT) has a much wider range of applicability and has a fundamental character, being based on the rigorous Kohn-Hohenberg theorem, at least in the static case [1]. However, DFT in nuclear physics involves quite a few open questions. There are still many different classes of functionals (local functionals like Skyrme ones, Gogny functionals and covariant functionals); in each class there is a proliferation of different parameter sets, and a strategy to build a universal Energy Density Functional (EDF) still need to be invented.

The time-dependent extension of DFT (TDDFT) is even more problematic, and at present it is realized only in the so-called adiabatic version. This means that the energy density at point $\mathbf{r}$ and time $t$ is assumed to depend only on the changes of the density at the same point and at the same time. On the other hand, the nuclear phenomenology is characterised by non-localities in space and time, and it is important to make an effort to include them on top of TDDFT. The particle-vibration coupling approach is one possibile way albeit of course not the only one. On top of the motion in a static, possibly non-local in space, mean-field, PVC takes into account the coupling with the mean-field fluctuations [2].

In this contribution, we wish to describe some of the recent calculations performed in our group along this line. Our starting point is one of the possible realisations of TDDFT, namely the self-consistent HartreeFock (HF) plus Random Phase Approximation (RPA)

\footnotetext{
ae-mail: colo@mi.infn.it
}

scheme based on Skyrme interactions, or functionals. We shall mainly discuss charge-exchange excitations like the Gamow-Teller resonances (GTRs), and for details we refer to Ref. [3], where our formalism has been introduced and illustrated. Some details that are common between chargeexchange and normal RPA can be found in Ref. [4], where HF-RPA for non charge-exchange models is thoroughly presented.

On top of RPA, we include PVC along the same line of the model that has been originally introduced in [3]. Such model has been revived and significantly extended in Refs. [5-7], where many approximations of the original implementation have been removed.

We apply this model to charge-exchange transitions like GT and $\beta$-decay in Sec. 3, after recalling the main features of the formalism in Sec. 2. In the last Sec. 4 we introduce a preliminary version of a model that is intended to implement the PVC idea to calculate the low-lying spectroscopy of odd nuclei, with an extension that is related to the inclusion of non-collective states.

\section{Formalism}

We employ the same formalism as in Refs. [5, 6], and here we only recall its essential points. We first carry out a self-consistent HF+RPA calculation of the GT strength, using a standard Skyrme interaction. Forward-going and backward-going amplitudes associated with the RPA eigenstates $|n\rangle$ will be denoted by $X_{p h}^{(n)}$ and $Y_{p h}^{(n)}$, respectively. Due to the effect of PVC, the RPA strength will be shifted and redistributed through the coupling to a set of doorway states, denoted by $|N\rangle$, made up with a particlehole ( $\mathrm{p}-\mathrm{h})$ excitation coupled to a collective vibration of angular momentum $J$. The properties of these collective 
vibrations, i.e. phonons $|n J\rangle$ are, in turn, obtained by computing the RPA response with the same Skyrme interaction, for states of natural parity $J^{\pi}=0^{+}, 1^{-}, 2^{+}, 3^{-}, 4^{+}$, $5^{-}$, and $6^{+}$. Only collective vibrations which absorb a fraction of the total isoscalar or isovector strength larger than $5 \%$ (and with energy less than $20 \mathrm{MeV}$ ) are included in the model space. This amounts to 22 phonons in the case, e.g., of the calculation performed for ${ }^{208} \mathrm{~Pb}$ with the interaction SkM*.

The GT strength is obtained from

$$
S(\omega)=-\frac{1}{\pi} \operatorname{Im} \sum_{v}\left\langle 0\left|\hat{O}_{\mathrm{GT}^{ \pm}}\right| v\right\rangle^{2} \frac{1}{\omega-\Omega_{v}+i\left(\frac{\Gamma_{v}}{2}+\Delta\right)},
$$

where $\hat{O}_{\mathrm{GT}^{ \pm}}$is the standard GT operator. $|v\rangle$ are the $1^{+}$ eigenstates of the system, associated with the complex eigenvalues $\Omega_{v}-i \frac{\Gamma_{v}}{2}$ and eigenvectors $\left(F^{(v)}, \bar{F}^{(v)}\right)$, that are obtained by diagonalizing the energy-dependent complex matrix

$\left(\begin{array}{cc}\mathcal{D}+\mathcal{A}_{1}(\omega) & \mathcal{A}_{2}(\omega) \\ -\mathcal{A}_{3}(\omega) & -\mathcal{D}-\mathcal{A}_{4}(\omega)\end{array}\right)\left(\begin{array}{c}F^{(v)} \\ \bar{F}^{(v)}\end{array}\right)=\left(\Omega_{v}-i \frac{\Gamma_{v}}{2}\right)\left(\begin{array}{c}F^{(v)} \\ \bar{F}^{(v)}\end{array}\right)$

Here, $\mathcal{D}$ is a diagonal matrix containing the positive RPA eigenvalues, and the $\mathcal{A}_{i}$ matrices are associated with the coupling to the doorway states. The expression of $\mathcal{A}_{i}$ in the RPA basis $|n\rangle$ reads

$\left(\mathcal{A}_{1}\right)_{m n}=\sum_{p h, p^{\prime} h^{\prime}} W_{p h, p^{\prime} h^{\prime}}^{\downarrow}(\omega) X_{p h}^{(m)} X_{p^{\prime} h^{\prime}}^{(n)}+W_{p h, p^{\prime} h^{\prime}}^{\downarrow *}(-\omega) Y_{p h}^{(m)} Y_{p^{\prime} h^{\prime}}^{(n)}$,

$\left(\mathcal{A}_{2}\right)_{m n}=\sum_{p h, p^{\prime} h^{\prime}} W_{p h, p^{\prime} h^{\prime}}^{\downarrow}(\omega) X_{p h}^{(m)} Y_{p^{\prime} h^{\prime}}^{(n)}+W_{p h, p^{\prime} h^{\prime}}^{\downarrow *}(-\omega) Y_{p h}^{(m)} X_{p^{\prime} h^{\prime}}^{(n)}$,

$\left(\mathcal{A}_{3}\right)_{m n}=\sum_{p h, p^{\prime} h^{\prime}} W_{p h, p^{\prime} h^{\prime}}^{\downarrow}(\omega) Y_{p h}^{(m)} X_{p^{\prime} h^{\prime}}^{(n)}+W_{p h, p^{\prime} h^{\prime}}^{\downarrow *}(-\omega) X_{p h}^{(m)} Y_{p^{\prime} h^{\prime}}^{(n)}$,

$\left(\mathcal{A}_{4}\right)_{m n}=\sum_{p h, p^{\prime} h^{\prime}} W_{p h, p^{\prime} h^{\prime}}^{\downarrow}(\omega) Y_{p h}^{(m)} Y_{p^{\prime} h^{\prime}}^{(n)}+W_{p h, p^{\prime} h^{\prime}}^{\downarrow *}(-\omega) X_{p h}^{(m)} X_{p^{\prime} h^{\prime}}^{(n)}$,

where $W^{\downarrow}$ is the matrix associated with the coupling of the particle-hole (p-h) states with the doorway states, that is,

$$
W_{p h, p^{\prime} h^{\prime}}^{\downarrow}(\omega)=\sum_{N} \frac{\langle p h|V| N\rangle\left\langle N|V| p^{\prime} h^{\prime}\right\rangle}{\omega-\omega_{N}+i \Delta} .
$$

The detailed expression of such matrix, together with its diagrammatic representation, can be found in Refs. [5, 6]. The averaging parameter $\Delta$ is introduced to avoid singularities in the denominator of Eq. (7), and a convenient value is, in practice, $\Delta=200 \mathrm{keV}$ or larger in the region of the GTR. A larger value of $\Delta$ can effectively take into account the coupling to more complex configurations (not included in the current model), and/or simulate the experimental resolution.

In the case of $\beta$-decay, we use the allowed GT approximation, namely we use the GT strength distribution in order to calculate the decay half-life. This distribution is obtained as a function of the excitation energy $E_{M}$ with respect to the mother nucleus, which is related to the energy $E$ in the daughter nucleus through the relation $E_{M}=E+\Delta B, \Delta B$ being the experimental binding energy difference between mother and daughter nucleus. The details of the $\beta$-decay calculation are described in Ref. [7]; here, we simply mention that we have found that the lifetimes converge for small values of $\Delta$, and we have chosen $\Delta=0.05 \mathrm{MeV}$ for the states in the $\beta$-decay window. The $\beta$-decay half-life is given by

$$
T_{1 / 2}=\frac{D}{g_{A}^{2} \int^{Q_{\beta}} S(E) f(Z, \omega) d E},
$$

where $f$ is the integrated phase space volume. The integration includes all the GT states with energy smaller than the $Q_{\beta}$ value. The value of $D$ is $6163.4 \mathrm{~s}$. The quenching effect is included in $g_{A}$ by taking $g_{A}=1.0$. In the GT strength distribution, we do not include any quenching factor. The integrated phase space volume reads

$$
f(Z, \omega)=\frac{1}{\left(m_{e} c^{2}\right)^{5}} \int_{m_{e} c^{2}}^{\omega} p_{e} E_{e}\left(\omega-E_{e}\right)^{2} F_{0}\left(Z+1, E_{e}\right) d E_{e},
$$

where $p_{e}, E_{e}$, and $F_{0}\left(Z+1, E_{e}\right)$ denote the momentum, energy and Fermi function of the emitted electron, respectively. $\omega$ is the energy difference between the initial and final nuclear state, and is connected with GT energy $E$ by $\omega=Q_{\beta}+m_{e} c^{2}-E$.

\section{Results and discussion}

\subsection{Gamow-Teller excitations}

In Fig. 1, the GT strength distributions of ${ }^{208} \mathrm{~Pb}$ calculated by RPA and RPA+PVC approach with the Skyrme interactions SkM* and SGII are shown. The excitation energies are given with respect to the parent nucleus. To compare with data, in panels (a) and (c) we adopt for the smearing parameter a value $\Delta=1 \mathrm{MeV}$, in both the RPA and RPA+PVC calculations. In panels (b) and (d) we also show the results obtained with a smaller value, that is, $\Delta=200 \mathrm{keV}$, as it allows to see the features of the theoretical GT distributions in more detail. With $\Delta=1 \mathrm{MeV}$, for both interactions the RPA+PVC model provides a good description of the line shape of the giant resonance. The essential effect coming from the inclusion of PVC is that the excitation energies are shifted downwards by $\approx 1-2 \mathrm{MeV}$, and in addition a conspicuous spreading width arises. Besides the main GT peak at $19.2 \mathrm{MeV}$, there is another low-energy peak produced by the RPA+PVC calculations, and located at about $11.8 \mathrm{MeV}$ (SkM*) or 12.5 $\mathrm{MeV}$ (SGII), which is not visible in the experiment.

Concerning strengths, we briefly discuss the case of the interaction $\mathrm{SkM}^{*}$ (SGII). The total $\mathrm{GT}^{-}\left[\sum B\left(\mathrm{GT}^{-}\right)\right]$ and $\mathrm{GT}^{+}\left[\sum B\left(\mathrm{GT}^{+}\right)\right]$strengths calculated in the RPA approach are 132.89 (132.95) and 0.90 (0.96), respectively. The RPA result for $\sum B\left(\mathrm{GT}^{-}\right)-\sum B\left(\mathrm{GT}^{+}\right)$exhausts $99.99 \%(99.99 \%)$ of the Ikeda sum rule. Only $1 \%(3 \%)$ of the calculated $\mathrm{GT}^{-}$strength lies at in the energy region above $25 \mathrm{MeV}$. In the RPA+PVC calculation, $\sum B\left(\mathrm{GT}^{-}\right)-\sum B\left(\mathrm{GT}^{+}\right)$exhausts $95.6 \%$ (95.2\%) of 

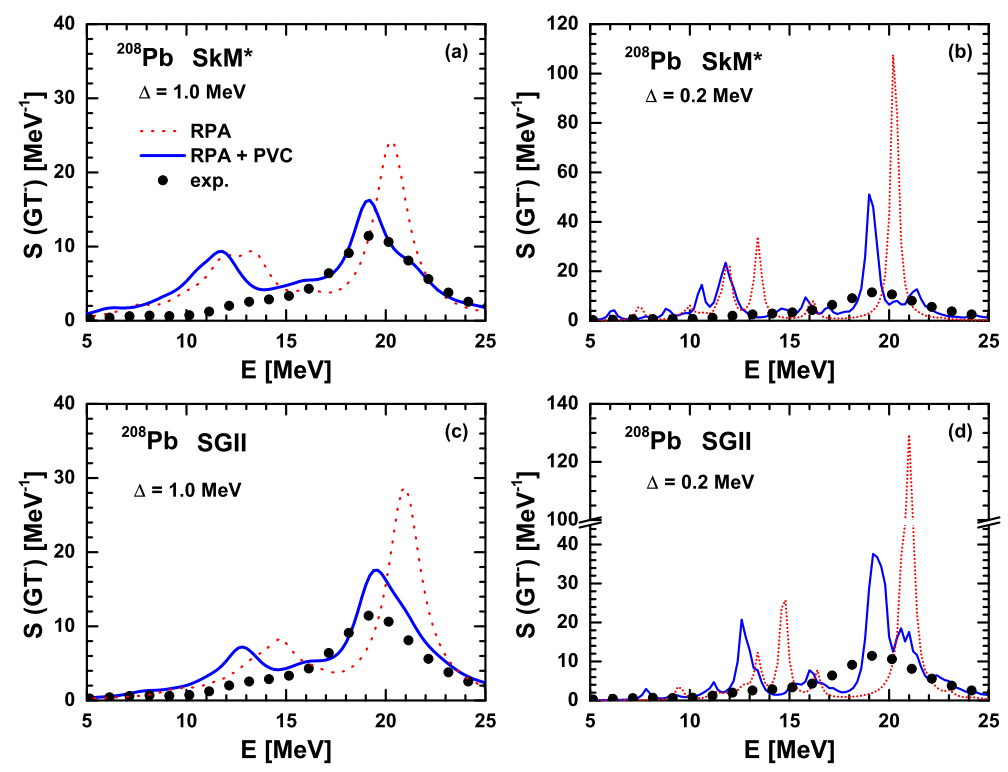

Figure 1. (Color online) Gamow-Teller strength distribution of ${ }^{208} \mathrm{~Pb}$ calculated by means of the RPA and RPA+PVC approaches with the Skyrme interactions SkM* and SGII, using the smearing parameter $\Delta=0.2$ and $1.0 \mathrm{MeV}$. The experimental data [8] are also shown for comparison. Figure adapted from Ref. [6].

the Ikeda sum rule up to the excitation energy of $60 \mathrm{MeV}$, while this value becomes $97.7 \%$ (97.3\%) in the case in which the smearing parameter $\Delta=0.2 \mathrm{MeV}$ is adopted. The experimental strength integrated up to $25 \mathrm{MeV}$ is about $68 \%$ (71\%) of the RPA+PVC result [8].

Previous studies found that RPA tensor correlations could shift about $10 \%$ of the sum rule to the excitation energy region above $30 \mathrm{MeV}[9,10]$. The inclusion of the coupling with the $\Delta$-isobar excitation could move strength to very high excitation energy, this amount being of the order of $10 \%$ of the total sum rule or less [11-13]. Concerning the remaining discrepancy with experiment, we cannot determine to which extent it can be attributed to deficiencies in the model, or to systematic uncertainties that the experiment was unable to pin down.

By reducing the value of the smearing parameter to $\Delta=200 \mathrm{keV}$, the detailed structure of the resonance is revealed. The main peak, which displayed a full width at half maximum (FWHM) of about $3.6 \mathrm{MeV}$ with $\Delta=1 \mathrm{MeV}$, is roughly split into two peaks. In the case of both interactions, but in particular for $\mathrm{SkM}^{*}$, more strength and a smaller width is obtained for the lower peak.

\section{$3.2 \beta$-decay}

In Fig. $2, \beta$-decay half-lives of ${ }^{78} \mathrm{Ni}$ calculated by RPA and RPA+PVC approaches with several different Skyrme interactions are compared with the experimental value [14]. The nucleus ${ }^{78} \mathrm{Ni}$ is a doubly magic one that is an important waiting point in the $r$-process, and represents a major bottleneck for the synthesis of heavier elements. The Skyrme interactions are not well constrained in the spinisospin channel, and as a consequence their predictions for the half-life can vary by more than two orders of magnitude. With the inclusion of PVC effects, the half-lives are

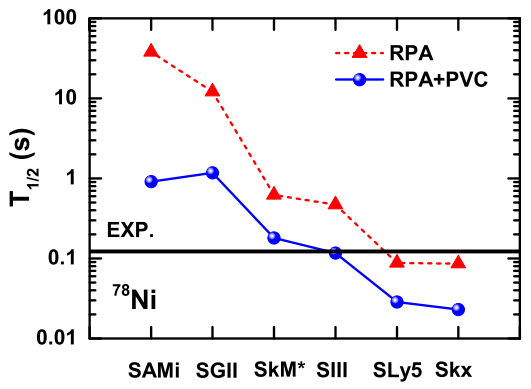

Figure 2. (Color online) $\beta$-decay half-life of ${ }^{78} \mathrm{Ni}$, calculated by RPA and RPA+PVC approaches with several Skyrme forces, in comparison with the experimental value [14]. Figure adapted from Ref. [7].

reduced for all interactions, and in particular the $\mathrm{SkM}^{*}$ and SIII results agree with the experimental value. It has to be stressed that the Skyrme force SkM* does not only reproduce well $\beta$-decay half-life, but also describes well the giant resonance line shape in ${ }^{208} \mathrm{~Pb}$ (as we have seen above) and in ${ }^{56} \mathrm{Ni}$ at the PVC level [5, 6]. Therefore, we shall use this interaction for further investigations. As we show and briefly discuss in Ref. [7], there may be some interaction, like SkX, that reproduces the $\beta$-decay half-lives at the level of RPA. However, we aim here at a description of these half-lives on equal footing not only with the centroid of giant resonances but also with their width and fragmentation.

In Fig. 3, we analyze how the PVC reduces the halflife. In panel (a), we present the GT strength distribution calculated by RPA and RPA+PVC model. The half-life is determined by the product of the strength with the phase space volume, which increases as the fifth power of the 


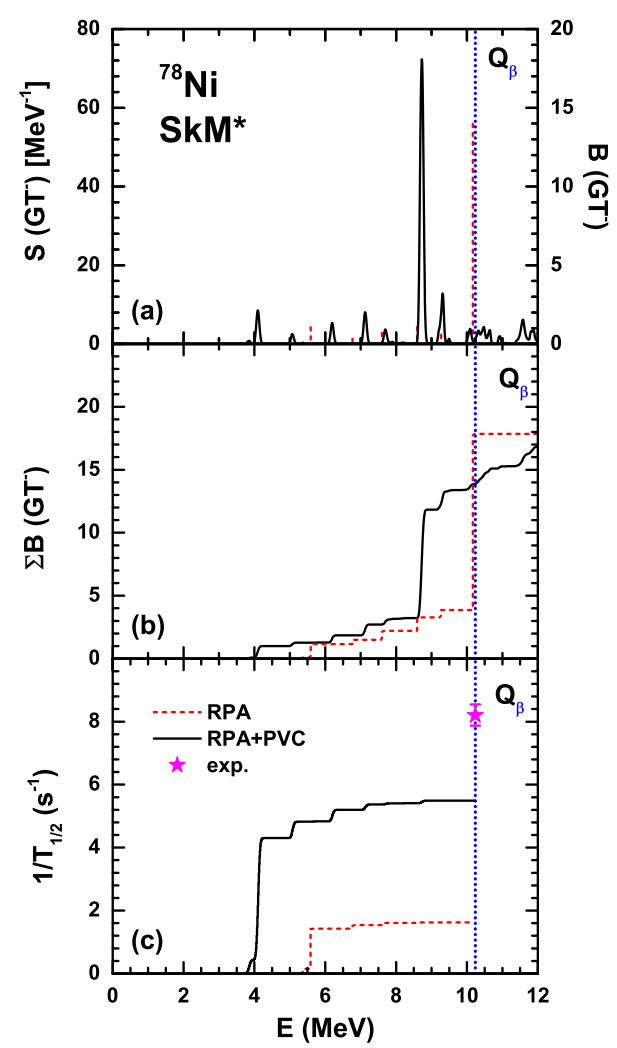

Figure 3. (Color online) The GT low-lying strength distribution with respect to daughter nucleus [panel (a)], the cumulative sum of the GT strength [panel (b)], and the cumulative sum of $1 / T_{1 / 2}$ [panel (c)]. The RPA and RPA+PVC results are shown respectively by the dotted line and solid line. The vertical dotted lines show the experimental value of $Q_{\beta}$ [15]. The experimental value of $1 / T_{1 / 2}[14]$ is indicated by the star. Figure adapted from Ref. [7].

energy, i.e., $f \propto\left(Q_{\beta}-E\right)^{5}$. So a little variation in excitation energy can induce a large variation in phase space volume, and as a consequence also in the half-life. With the inclusion of PVC, the RPA strength is shifted downwards by about $2 \mathrm{MeV}$. This causes a big increase in phase space volume, and in turn, a big decrease in the half-life. In panel (b), the cumulative sums of the GT strength calculated by RPA and RPA+PVC model are shown. The strength of each peak is basically kept conserved as in the RPA case after the inclusion of PVC effect. In panel (c), the cumulative sums of $1 / T_{1 / 2}$ calculated by RPA and RPA + PVC model are shown. With the inclusion of PVC, the $1 / T_{1 / 2}$ increases considerably as compared to the RPA result, and gets quite close to the experimental value. So in conclusion, although the PVC does not change the strength of each peak, it reduces the half-life dramatically by shifting downwards the excitation energy.

Finally, the lifetimes of the four nuclei ${ }^{132} \mathrm{Sn},{ }^{68} \mathrm{Ni}$, ${ }^{34} \mathrm{Si}$, and ${ }^{78} \mathrm{Ni}$ are compared with experiment in Fig. 4 in the case of the SkM* force. The RPA results markedly overestimate the half-life for all nuclei. Except for ${ }^{78} \mathrm{Ni}$, the discrepancy amounts to more than one order of magnitude. For ${ }^{132} \mathrm{Sn}$, the RPA model even gives an infinite half-life, i.e., the nucleus is stable. The effect of the PVC

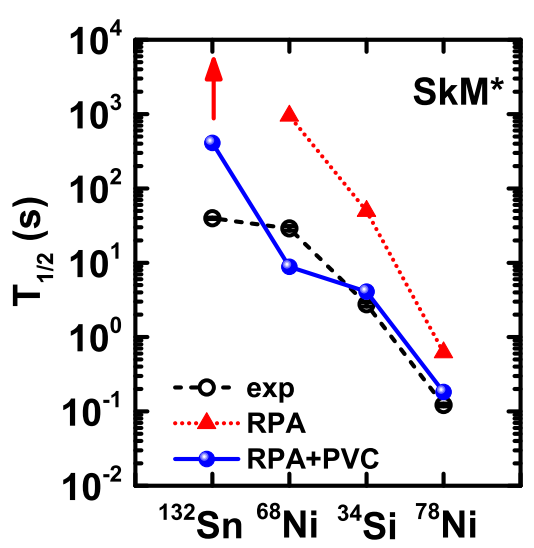

Figure 4. (Color online) The $\beta$-decay half-lives of ${ }^{132} \mathrm{Sn},{ }^{68} \mathrm{Ni}$, ${ }^{34} \mathrm{Si}$, and ${ }^{78} \mathrm{Ni}$, calculated by RPA and RPA+PVC approaches with the interaction $\mathrm{SkM}^{*}$, respectively, in comparison with experimental values $[14,15]$. The arrow denotes an infinite halflife. Figure adapted from Ref. [7].

decreases the values of $T_{1 / 2}$ by large factors compared to RPA, substantially improving the overall agreement with experimental data.

\section{A model for odd nuclei}

Odd nuclei are also amenable to a PVC description (see also [16] and references therein). The standard weakcoupling model [17] has been employed by some of us to describe some of the low-lying states around the ${ }^{48} \mathrm{Ca}$ core $[18,19]$. The low-lying spectra in odd nuclei like ${ }^{47,49} \mathrm{Ca}$, however, show the presence of states with different character. Some of them have mainly particle-like (or hole-like) character, and are characterised by large spectroscopic factors in pick-up (or stripping) reactions ${ }^{1}$. Some others may come from the coupling of the particle (or hole) with a core vibration. We take as a signature of such character the fact that the decay reduced transition probability from these states to the odd nucleus ground state should be similar to the reduced transition probability of the core vibration. In fact, in the weak coupling scheme these reduced transition probabilities are equal, that is,

$$
B\left(E \lambda,\left[j^{\prime} \otimes \lambda\right]_{j} \rightarrow j^{\prime}\right)=B(E \lambda, \lambda \rightarrow 0) .
$$

At the same time, there might be further states that have for instance $2 \mathrm{p}-1 \mathrm{~h}$ (or $1 \mathrm{~h}-2 \mathrm{p}$ ) character and do not fit the weak-coupling PVC scheme by definition. These states appear, for instance, in shell model calculations whose results have been displayed in Ref. [19]; at the same time, it is well known that such shell-model calculations have difficulties to pin down the particle-vibrational states, and are too demanding or simply impossible as mass number and/or excitation energy increase.

\footnotetext{
${ }^{1}$ We are well aware of the problems associated with a clean definition of spectroscopic factors. However, such problems do not prevent from using spectroscopic factors for a qualitative indication about the nature of observed states discussed here.
} 
All these considerations have motivated the formulation of a hybrid model that we shall call Hybrid Configuration Mixing (HCM) model in what follows. In this model, we include particle states together with states that for practical reasons emerge from RPA calculations, but can have either collective character or pure $\mathrm{p}$-h nature. In either case, we should of course account at our best for Pauli principle violations, but the problem is certainly more severe in the case of pure p-h states. Some of us are currently employing this model to explain the spectra of odd nuclei with one particle outside a magic core, like ${ }^{133} \mathrm{Sb}$.

In this model, we solve the Hamiltonian

$$
\begin{gathered}
H=H_{0}+V, \\
H_{0}=\sum_{j m} \varepsilon_{j} a_{j m}^{\dagger} a_{j m}+\sum_{N J M} \hbar \omega_{N J} \Gamma_{N J M}^{\dagger} \Gamma_{N J M}, \\
V=\sum_{j m j^{\prime} m^{\prime}} \sum_{N J M} h\left(j m ; j^{\prime} m^{\prime}, N J M\right) a_{j m}\left[a_{j^{\prime}}^{\dagger} \otimes \Gamma_{N J}^{\dagger}\right]_{j m},
\end{gathered}
$$

where the single particle states of the core nucleus are labelled for simplicity by $\mathrm{jm}$ instead of $\mathrm{nlj} m$ and have energies $\varepsilon_{j}$, whereas the phonons with angular momentum $J M$ are labelled by the index $N$ and have energies $\hbar \omega_{N J}$. As usual, $a^{+}\left(\Gamma^{+}\right)$refer to single-particle states (RPA states) creation operator. If we use consistently the Skyrme interaction, these states emerge from HF and RPA calculations, respectively; in this case, the matrix elements $h$ have the expression that can be read from Eq. (A1) of Ref. [20].

The Hamiltonian (4) can be diagonalised separately in different Hilbert subspaces with good angular momentum $j$ and parity $\pi$. The basis states in these subspaces are either particle states,

$$
|j m\rangle=a_{j m}^{\dagger}|0\rangle,
$$

where $|0\rangle$ is the even-even core, or coupled states

$$
\left|\left[j^{\prime} \otimes N J\right]_{j m}\right\rangle
$$

that, as discussed above, can either be particles plus genuine collective states, or pure $2 \mathrm{p}-1 \mathrm{~h}$ states. This ensemble will define the basis for the calculation of the $\mathrm{A}+1 \mathrm{nu}-$ cleus close to a core nucleus A. The model space could be in principle extended if needed. The matrix elements between the configurations (12) and (13) are $\left\langle j\|V\| j^{\prime} J\right\rangle / \hat{j}$ [cf. Eq. (A9) of [20]]. So, the Hamiltonian in each of the subspaces has the typical form

$$
H=\left(\begin{array}{cc}
\varepsilon_{n_{1} l j} & \frac{\left\langle n_{1} l j\|V\| n_{1}^{\prime} l_{1}^{\prime} j_{1}^{\prime} N_{1} J_{1}\right\rangle}{\hat{j}} \\
\frac{\left\langle n_{1} l j\|V\| n_{1}^{\prime} l^{\prime} j_{1}^{\prime} N_{1} J_{1}\right\rangle}{\hat{j}} & \varepsilon_{n_{1}^{\prime} l_{1}^{\prime} j_{1}^{\prime}}+\hbar \omega_{N_{1} J_{1}}
\end{array}\right) .
$$

We can label the basis states [either of the type (12) or (13)] with $|i\rangle$, so that the above matrix is $H_{i i^{\prime}}$.

The main problem is that the basis in the second subspace [the one spanned by the states (13)] may be non orthogonal and overcomplete. This problem by using the technique of Ref. [21]. For this aim, we need the overlap matrix $N_{i i^{\prime}}$ between the basis states in the Hilbert subspace under study. This matrix is clearly diagonal in the subspace of states (12), and zero if matrix elements between states of the type (12) and (13) are considered. On the other hand, we do have non-trivial overlaps between states of the type (13). Such overlaps can be calculated if the structure of the states emerging from the RPA calculation is known, and the detailed formulas will be displayed in forthcoming publications. The eigenvalue problem becomes of the generalised type, that is,

$$
(H-E N) X=0 \text {, }
$$

where $E$ labels a generic eigenvalue and $X$ is the corresponding eigenvector. We plan to obtain results for different multipoles in few nuclei, and comparisons with other existing schemes like the one of Ref. [22] will be carried out.

\section{Conclusions}

There are recent developments in self-consistent PVC calculations that employ Skyrme interactions, and we have described some of them in the present contribution. Of course, such attempts complement our understanding of the limitations of currently used Skyrme functionals, and may pave the way for more advanced and accurate manybody schemes.

PVC calculations for giant resonances overcome one of the main limitations of RPA, which cannot describe the spreading width. In the case of the Gamow-Teller resonance of ${ }^{208} \mathrm{~Pb}$, it has been found that the PVC shifts the excitation energy downwards by $1-2 \mathrm{MeV}$, and induces a non-negligible spreading of the strength of the main RPA peak. Good agreement with the experimental GT resonance line shape can be obtained, at least for some Skyrme functionals as SkM*.

The downward shift of the strength is of particular importance in the calculation of beta-decay half-lives, which are more sensitive to the position of the GT states and to the consequent increase in phase space, rather than to the integrated strength below $Q_{\beta}$, which is not much changed by the inclusion of PVC. The RPA+PVC model reduces the $\beta$-decay half-lives of the nuclei ${ }^{132} \mathrm{Sn},{ }^{68} \mathrm{Ni},{ }^{34} \mathrm{Si}$, and ${ }^{78} \mathrm{Ni}$ (all treated as magic). The $\mathrm{SkM}^{*}$ functional leads to a good agreement with experiment both for the strength distribution in the GTR region and for the $\beta$-decay lifetimes.

The PVC plays an important role in the study of the low-lying spectroscopy of odd nuclei. We wish to describe the low-lying states of the $\mathrm{A}+1$ nucleus close to the core $\mathrm{A}$ as combinations of particle states, and particle+RPA coupled states, that can have either particle-vibrational character or $2 p-1 h$ nature. Preliminary results are already available for ${ }^{133} \mathrm{Sb}$

\section{Acknowledgements}

The NSRT15 conference has celebrated the anniversary of Prof. V.G. Soloviev. As a founder of the Dubna school, he has been the main contributor of the so-called Quasiparticle Phonon Model (QPM), and within such framework many calculations that have been performed are similar in spirit to what we have discussed above, the main difference being the choice of self-consistency in our case. It is 
a pleasure to acknowledge the huge contribution to nuclear structure physics by Prof. V.G. Soloviev, and the many discussions and collaborations with members of the group he has started and directed.

\section{References}

[1] G. Colò, C. Barbieri, D. Vretenar, 2015, to be published.

[2] C. Mahaux, P.-F. Bortignon, R.A. Broglia, C.H. Dasso, Phys. Rep. 4, 1 (1985)

[3] G. Colò, N. Van Giai, P.-F. Bortignon, R.A. Broglia, Phys. Rev. C 50, 1496 (1994)

[4] G. Colò, L. Cao, N.V. Giai, L. Capelli, Computer Phys. Communications 184, 142 (2013)

[5] Y.F. Niu, G. Colò, M. Brenna, P.-F. Bortignon, J. Meng, Phys. Rev. C 85, 034314 (2012)

[6] Y.F. Niu, G. Colò, E. Vigezzi, Phys. Rev. C 90, 054328 (2014)

[7] Y.F. Niu, Z.M. Niu, G. Colò, and E. Vigezzi, Phys. Rev. Lett. 114, 142501 (2015)

[8] T. Wakasa, M. Okamoto, M. Dozono, K. Hatanaka, M. Ichimura, S. Kuroita, Y. Maeda, H. Miyasako, T. Noro, T. Saito, et al., Phys. Rev. C 85, 064606 (2012)

[9] C.L. Bai, H. Sagawa, H.Q. Zhang, X.Z. Zhang, G. Colò, F.R. Xu, Phys. Lett. B 675, 28 (2009)

[10] C.L. Bai, H.Q. Zhang, X.Z. Zhang, F.R. Xu, H. Sagawa, G. Colò, Phys. Rev. C 79, 041301 (2009)

[11] B.A. Brown, B.H. Wildenthal, Nucl. Phys. A 474, 290 (1987)
[12] T.Wakasa, H. Sakai, H. Okamura, H. Otsu, S. Fujita, S. Ishida, N. Sakamoto, T. Uesaka, Y. Satou, M.B. Greenfield, et al., Phys. Rev. C 55, 2909 (1997)

[13] K. Yako, H. Sakai, M. Greenfield, K. Hatanaka, M. Hatano, J. Kamiya, H. Kato, Y. Kitamura, Y. Maeda, C. Morris, et al., Phys. Lett. B 615, 193 (2005)

[14] Z.Y. Xu, S. Nishimura, G. Lorusso, F. Browne, P. Doornenbal, G. Gey, H.S. Jung, Z. Li, M. Niikura, P.A. Söderström, et al., Phys. Rev. Lett. 113, 032505 (2014)

[15] M. Wang, G. Audi, A.H. Wapstra, F.G. Kondev, M. MacCormick, X. Xu, B. Pfeiffer, Chin. Phys. C 36, 1603 (2012)

[16] A. Idini, G. Potel, F. Barranco, E. Vigezzi, R.A. Broglia, Phys. Rev. C 92, 031304 (2015)

[17] A. Bohr, B.M. Mottelson, Nuclear Structure. Vol. II: Nuclear Deformations (W.A. Benjamin, New York, 1980)

[18] D. Montanari, S. Leoni, D. Mengoni, G. Benzoni, N. Blasi, G. Bocchi, P. Bortignon, A. Bracco, F. Camera, G. Colò, et al., Phys. Lett. B 697, 288 (2011)

[19] D. Montanari, S. Leoni, D. Mengoni, J.J. ValienteDobon, G. Benzoni, N. Blasi, G. Bocchi, P.-F. Bortignon, S. Bottoni, A. Bracco, et al., Phys. Rev. C 85, 044301 (2012)

[20] G. Colò, H. Sagawa, P.-F. Bortignon, Phys. Rev. C 82, 064307 (2010)

[21] D.J. Rowe, J. Math. Phys. 10, 1774 (1969)

[22] S. Mishev, V.V. Voronov, Phys. Rev. C 78, 024310 (2008) 